\title{
BOAT-BASED MOBILE LASER SCANNING FOR SHORELINE MONITORING OF LARGE LAKES
}

\author{
D. Schneider ${ }^{1}$, R. Blaskow ${ }^{1}$ \\ ${ }^{1}$ TU Dresden, Institute of Photogrammetry and Remote Sensing, Germany - (danilo.schneider, robert.blaskow)@tu-dresden.de
}

\author{
Commission II, WG II/10
}

KEY WORDS: shoreline monitoring, mobile laser scanning (MLS), terrestrial laser scanning, UAV, structure-from-motion (SfM)

\begin{abstract}
:
In many applications, it is necessary to measure the shore areas of water bodies in three dimensions, for example for the planning of shore protection measures or regularly for water body monitoring. The complete recording of long shorelines can be very timeconsuming with conventional methods. This paper presents the surveying of two lakes using a mobile laser scanner system. In order to close non-visible areas, the measurement was supplemented with terrestrial laser scanning and with UAV-supported photogrammetric methods. The result is an almost complete point cloud of the shoreline, each more than $8 \mathrm{~km}$ long, with a point spacing of a few millimetres.
\end{abstract}

\section{INTRODUCTION}

The Lausitzer Seenland is an artificially created lake district, which is developed by flooding disused coalmines. It consists of 23 post-mining lakes and the total water area amounts to $14^{\prime} 000$ hectare. The area is planned to be developed from a mining region to a modern touristic region. By this means, it aims to become Europe's largest artificial water landscape until 2030.

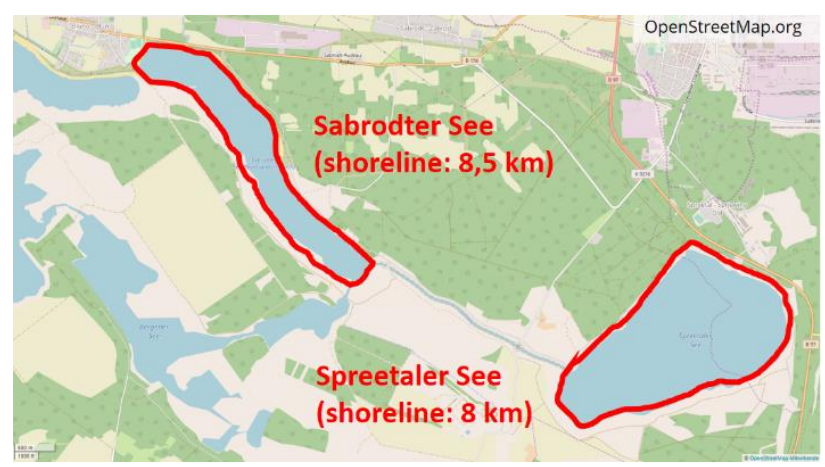

Figure 1. Study area: shoreline of two lakes

As the flooding is still in progress, there are ongoing dynamic changes such as local landslides. Therefore, it is essential to monitor the shorelines regularly. Together with the operating company, the Lausitzer und Mitteldeutsche BergbauVerwaltungsgesellschaft $\mathrm{mbH}$, two lakes have been chosen exemplary to investigate the suitability and efficiency of mobile laser scanning. The shoreline of both lakes together has an extent of more than $16 \mathrm{~km}$ (Fig. 1). The flooding of these lakes started approximately 10 years ago and is partly still in progress. Furthermore there are still construction activities such as compaction blasting.

Although limited to smaller regions, similar investigations with a boat-based mobile laser scanner system have been already described in (Mohamed, 2007 and Vaaja et al., 2013).

\section{METHOD}

Conventional 3D acquisition methods of shore areas are airborne laser scanning (ALS) or total station measurements. Airborne laser scanning is able to cover large areas in short time, but it is too expensive and inflexible for periodic monitoring. Although total station measurements are very accurate, it requires entering the partly dangerous shore regions, which is often not allowed due to the risk of landslides or subsidence. Another practical limitation for the measurement of the water shoreline is bankside vegetation. Thus, a survey of the whole shoreline solely using terrestrial laser scanning (TLS) or UAV-based structure-frommotion (SfM) is not practicable for such lakes.
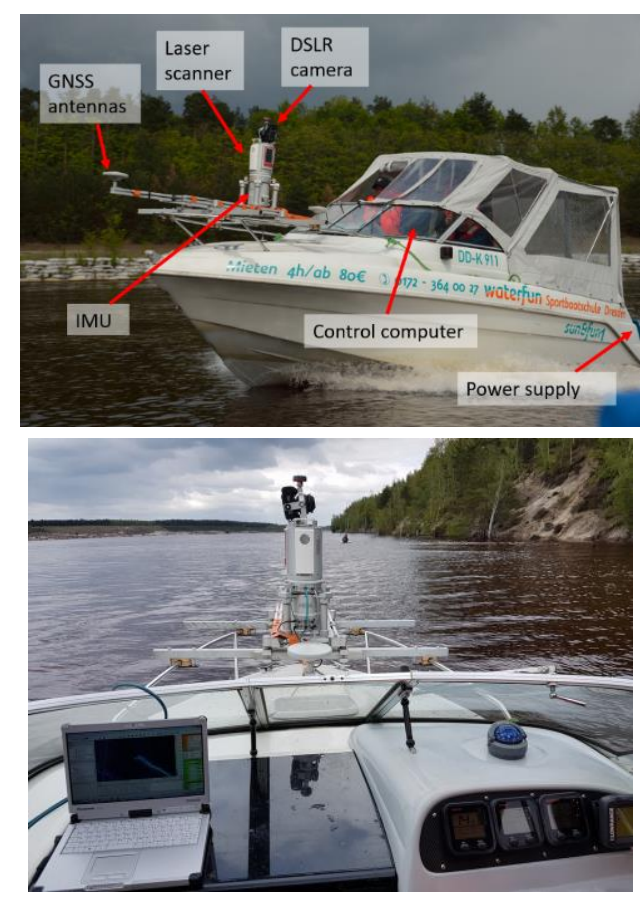

Figure 2. Mobile laser scanner system and its components courtesy of RIEGL Laser Measurement Systems GmbH 
For these reasons, a mobile laser scanner system RIEGL VMZ was applied to acquire a high-density point cloud of the shorelines of the two lakes (Fig. 2). The system consists of an Applanix IMU/GNSS unit with an absolute position accuracy (world coordinates) in dynamic mode of $2-5 \mathrm{~cm}$ and an angular accuracy of approx. $0.01^{\circ}$ to continuously determine the position and orientation of the RIEGL VZ-2000i laser scanner which is set into the 2D profile measurement mode (Fig. 3). Additionally a DSLR camera is mounted on top of the scanner, which acquires images every 2 seconds while the scanner is running. As the exterior orientation of the camera with respect to the scanner was calibrated, the imagery allows for colorization of the point cloud and supports the interpretation of the recorded point cloud data.

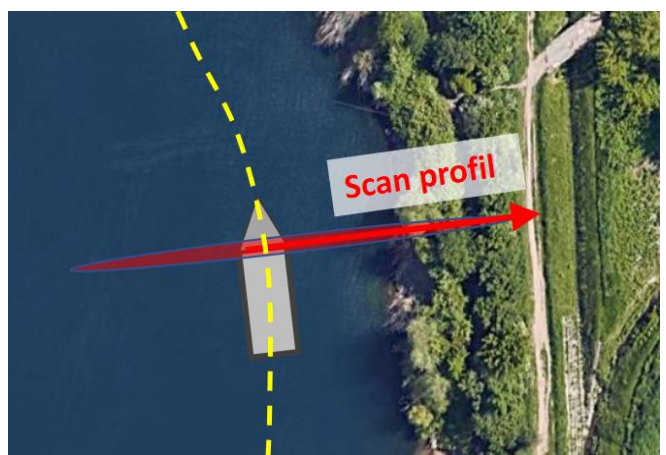

Figure 3. Procedure: boat trajectory and 2D scan profile

The laser scanner itself has a maximum range of $2.5 \mathrm{~km}$ at an effective measurement rate of 21,000 points per second and a surface reflectivity of $90 \%$. If a higher scanning speed is set to achieve a higher point density on the shoreline surface, the maximum distance decreases accordingly. In this specific case, the two lakes were recorded at a measuring rate of 500,000 points per second. Depending on the reflectivity of the shoreline surface, the maximum range varies between 300 and $600 \mathrm{~m}$.

The recording was carried out at walking speed $(6 \mathrm{~km} / \mathrm{h})$ at a distance of 50 to $100 \mathrm{~m}$ from the shoreline. Including calibration of the system, the survey took about 2 hours for one of the two lakes.

The post-processing utilizes two RIEGL software tools (RiPROCESS and RiWORLD) and consist of different steps as geo-referencing and the refinement of the trajectory based on the overlap of the start and end point of the trajectory. Moreover, the so-called multiple-time-around (MTA) zones had to be resolved.

\section{LIMITATIONS}

Although the scanning from the lake's perspective mostly allows for capturing most parts of the shoreline there are some limitations (Fig. 4). First, there are occlusion due to relatively high shorelines, which do not allow scanning the surface behind it. Second, there are further occlusions due to vegetation within the water such as reed. Both problems result in large gaps in the point cloud as shown in Fig. 5 and 6.
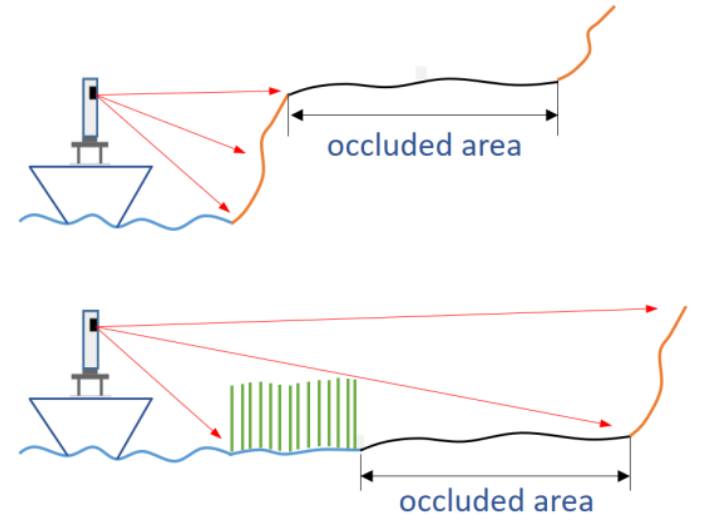

Figure 4. Occlusions due to specific shoreline geometry (top) and vegetation (bottom).

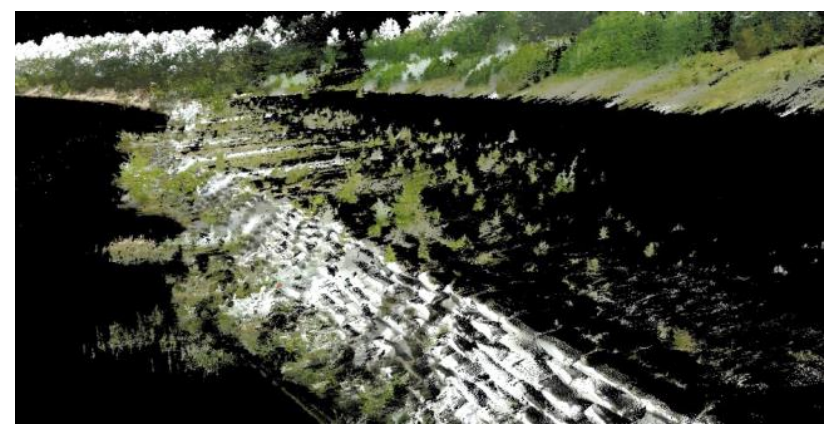

Figure 5. Gaps within the data due to shoreline geometry

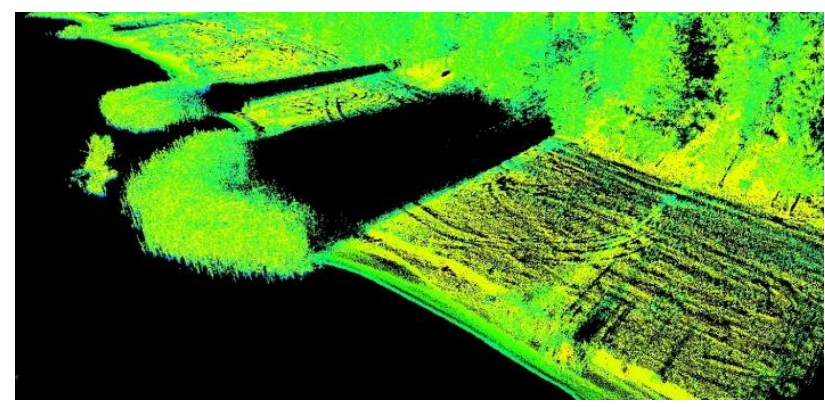

Figure 6. Gaps due to occlusions caused by vegetation

\section{ADDITIONAL MEASUREMENTS}

In order to close these gaps in the data the measurements from the boat were complemented with terrestrial laser scanning (Fig. 7 and 8) and UAV photogrammetry (Fig. 9 and 10) as well. Five sub-areas per lake have been chosen for measuring with both methods.

A laser scanner RIEGL VZ-400i was used with 5 to 10 scanning positions in each sub-area. For the UAV photogrammetry a DJI drone was utilized which flew in a height between 25 and $50 \mathrm{~m}$ above the ground. The flight in each sub-area took approximately 10 minutes and resulted in an image block of up to 170 images. 


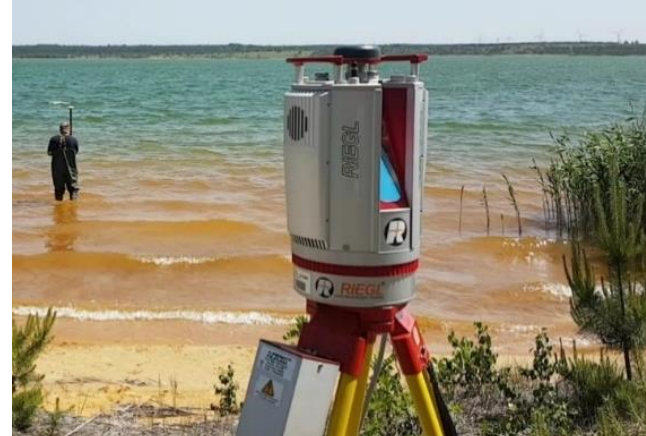

Figure 7. Terrestrial laser scanning with a RIEGL VZ400i

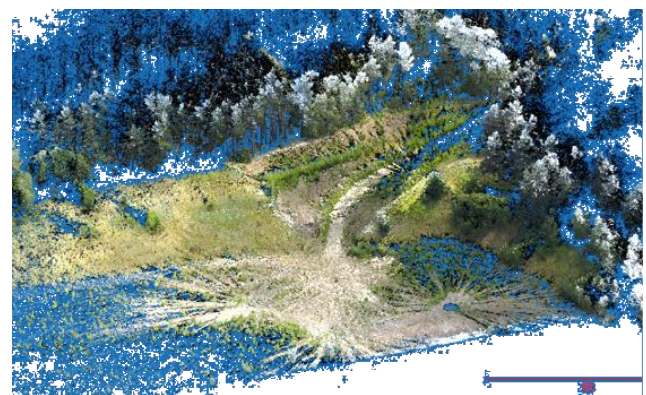

Figure 8. Terrestrial laser scanning point cloud of one of five sub-areas, scanned from 10 laser scanner positions

The post-processing of the terrestrial laser scanner data consists of point cloud registration, geo-referencing based on control points, point cloud filtering and sub-sampling.

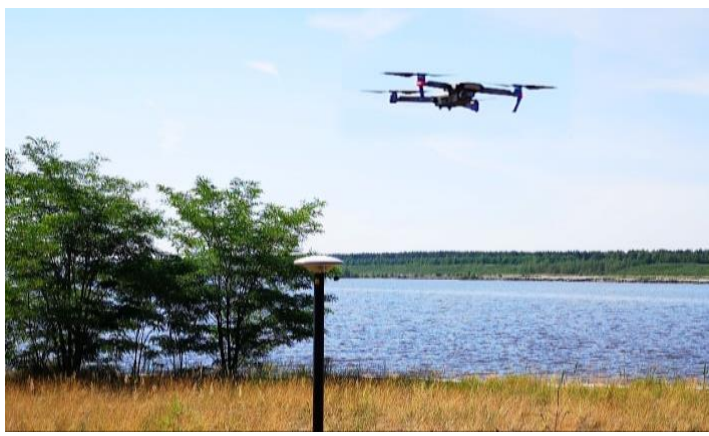

Figure 9. UAV flight with a DJI Mavic Pro Platinum

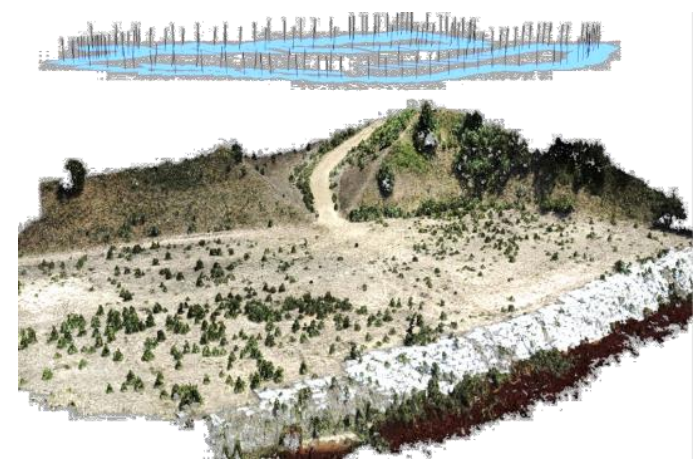

Figure 10. SfM point cloud of one of five sub-areas
The UAV imagery has been processed with the widely-used commercial structure-from-motion software Agisoft Metahape. The SfM pipeline includes automatic feature point detection, image orientation and dense image matching.

Scaling and geo-referencing was initially performed based on the on-board GNSS system.

\section{RESULTS}

The result was a point cloud of about 15 billion points per lake (Fig. 4) with the attributes intensity and reflectivity as well as colour (RGB) recorded with the Nikon D810 mounted on top of the scanning system. The average point distance is in the lower $\mathrm{mm}$ range, depending on the current measuring distance. The absolute accuracy in a world coordinate system (ETRS89) is in the range of $5 \mathrm{~cm}$. However, the relative accuracy is much higher $(2-5 \mathrm{~mm})$, which is depicted with a section of the point cloud in Fig. 11 showing an access ramp to the former coalmine destroyed by a local landslide.

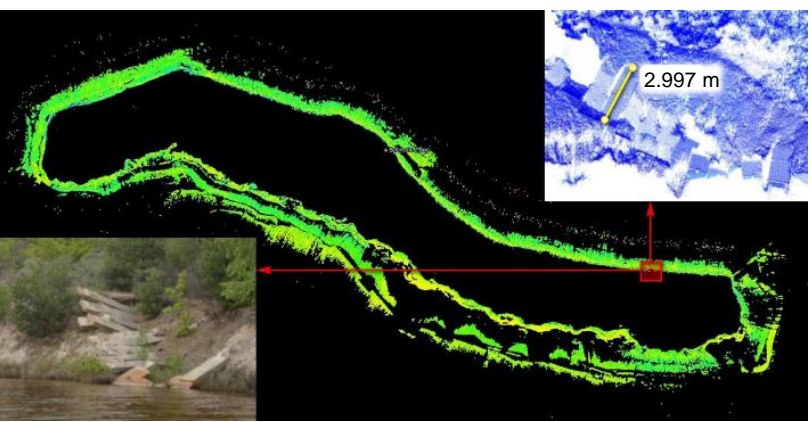

Figure 11. Point cloud of the shoreline of the Sabrodter See

The following figures show the final result after data fusion (Fig 12 and 13). The green areas are the sub-areas measured additionally with TLS and UAV photogrammetry.

Finally it can be summarised that the requirements defined in advance have been fulfilled: approx. 16 billion points per lake were captured in short time. The results have the required resolution, precision and completeness, although only after fusion with additional point clouds.

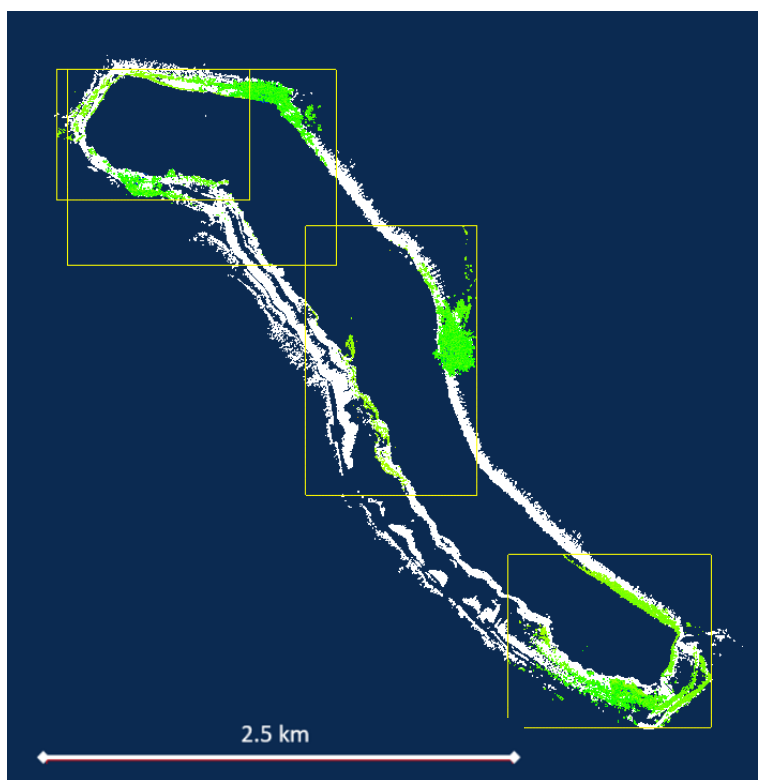

Figure 12. Final point clouds of Sabrodter See, with additional TLS and UAV-SfM point clouds in green 


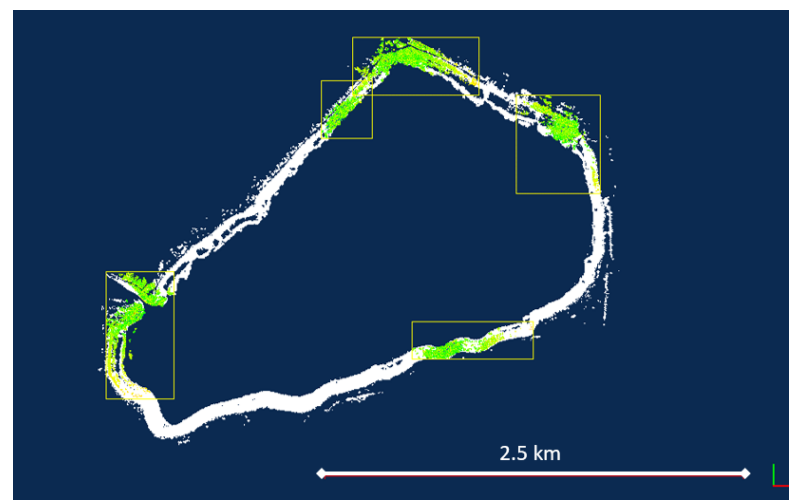

Figure 13. Final point clouds of the Spreetaler See, with additional TLS and UAV-SfM point clouds in green

\section{VALIDATION AND DISCUSSION}

As the additional data has also some overlap, it is possible to use these additional data for validation comparisons as well.

In order to validate the MLS data the differences to the TLS data as reference (which were registered via control points measured with RTK-GNSS) were computed. This comparison only resulted in random errors below $5 \mathrm{~cm}$, which confirms the manufacturer's data and validates the precision and reliability of the MLS point cloud.

Furthermore the UAV results were compared against the combined laser scanning point cloud, which resulted in significant systematic errors. The combined laser scanning point cloud (TLS+MLS) and the UAV-SfM point cloud of the same area shown in Fig. 8 and Fig. 10 were compared to each other. After an automatic registration in the software CloudCompare applying an ICP algorithm both point clouds were overlaid and subsequently the cloud-to-cloud distances (C2C) were calculated, which is shown in Fig. 14.

These cloud-to-cloud distances are visualised with different colours: blue means only a few centimetres, green is approximately $1 \mathrm{~m}$ and orange or red means a difference up to 2 $\mathrm{m}$ and above.

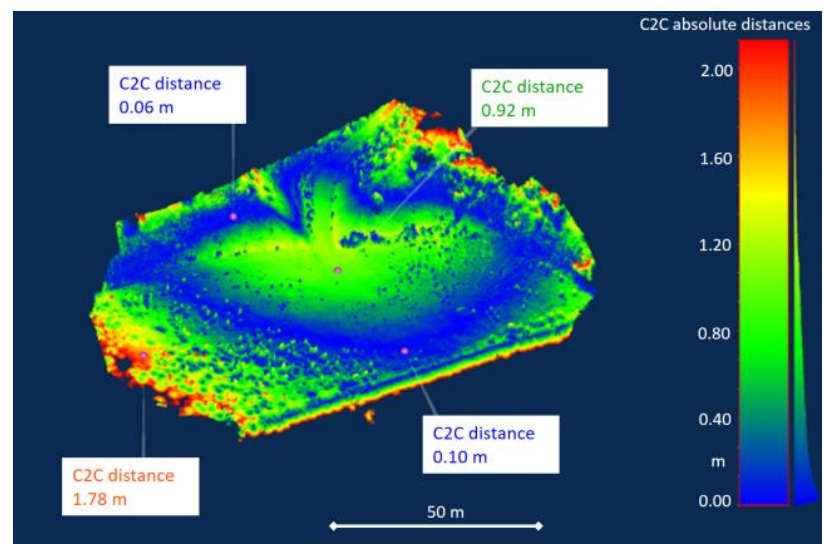

Figure 14. Cloud-to-cloud distances of the UAV-SfM to the laser scanning point cloud for one sub-area (CloudCompare)

There are obvious systematic effects. The characteristic of this systematic effect clearly indicates that it comes from the UAV point cloud. This effect is already described and named "dome effect" in the literature (e.g. Eltner \& Schneider, 2015).
The reason for this dome effect is the parallel-axes image configuration and the SfM-processing without the support of additional ground control points.

This shows that UAV generated point clouds or 3D-models should not be used without a thorough accuracy check.

\section{SUMMARY}

This paper has shown an efficient way of surveying the shorelines of small and medium-sized lakes. The boat-based MLS method presented here provides a highly accurate and almost complete bank representation and allows for periodical documentation. It is not necessary to enter dangerous or inaccessible bank areas. The viewing direction from the lake to the shoreline is more suitable in comparison to airborne methods. The data acquisition takes only short time (for example 4 lakes in one day). The results are a good basis for infrastructural planning, topographic monitoring or for the simulation of different scenarios (flooding, runoff behaviour). For this purpose, the generated point clouds can be processed in a variety of ways. For example, a raster representation can be interpolated or a closed surface mesh can be generated by triangulation. In addition to the visualisation of the surface for documentation purposes, which allows the sampling of discrete dimensions, any 2D profiles of the bank areas can be generated.

There are also some limitations as the big amount of data, which requires a lot of post-processing time and in case of occlusions, it might be useful to supplement the data with other surveying methods as presented. It could be shown that the gaps contained in the MLS scan can be closed using TLS and UAV photogrammetry.

Altogether boat-based MLS data provide an ideal basis for a wide range of planning and monitoring applications.

\section{ACKNOWLEDGEMENTS}

The authors would like to thank the following companies or institutions for their kind support:

The Lausitzer und Mitteldeutsche Bergbau-Verwaltungsgesellschaft for organising access to the two lakes, the company RIEGL Laser Measurement systems for providing the equipment and supporting the data processing, the Waterfun Sportbootschule Dresden for renting the boat. The project was jointly carried out together with the Institute of Hydraulic Engineering and Technical Hydromechanics, Technische Universität Dresden.

\section{REFERENCES}

Eltner, A., Schneider, D., 2015: Analysis of Different Methods for 3D Reconstruction of Natural Surfaces from Parallel-Axes UAV Images. The Photogrammetric Record, 30(151), p. 279299.

Mohamed, A.H., 2007: Scanning the Waters. POB - Point of Beginning. https://www.pobonline.com/articles/91477-scanning -the-waters (30 January 2021).

Riegl VMZ Infosheet, 2018, riegl.com/uploads/tx_pxpriegldo wnloads/RIEGL_VMZ_at-a-glance_brochure_2018-11-28.pdf (30 January 2021)

Vaaja, M., Kukko. A., Kaartinen, H., Kurkela, M., Kasvi, E., Flener, C., Hyyppä, H., Hyyppä, J., Järvelä, J., Alho, P., 2013: Data Processing and Quality Evaluation of a Boat-Based Mobile Laser Scanning System. Sensors 2013, 13, 12497-12515; doi:10.3390/s130912497 